\title{
Techniques for the rapid and sensitive detection of penicillinase
}

\author{
R. J. HOLT AND G. T. STEWART \\ From Queen Mary's Hospital for Children, Carshalton, Surrey
}

SYNOPSIS Practical problems in the detection of penicillinase are discussed. A membrane technique for this purpose is described, with various modifications, suitable for screening large numbers of organisms and yet more sensitive than most methods.

A one-stage membrane technique is adequate for detecting hydrolysis of penicillin $\mathrm{G}$ but a twostage technique is required to demonstrate hydrolysis of some other penicillins. A gradient adaptation can be used for quantitative purposes.

Staphylococci, coliforms, Proteus, and other organisms can be tested in this way for penicillinase formation; the method is also suitable for testing lysates, filtrates, and dialysates, provided independent provision is made for enzyme inducement.

The production by various bacteria of an enzyme capable of inactivating penicillin was first reported by several workers in the early 1940 s. Their views as to the endocellular or exocullular nature of this enzyme differed considerably, and were conditioned largely by the methods used for its detection and separation. At this time two basic techniques were in use. One was the direct destruction of penicillin in liquid by the addition of a whole culture filtrate, or supernatant, of the test organism. The detection was completed by the subsequent assay of loss of penicillin activity, using a suitably sensitive indicator organism. This followed the original suggestion of Abraham and Chain in 1940. A disc variation was also suggested, the test organism being spotted on to discs impregnated with dilute penicillin solution. The discs were placed on pavement inocula on agar plates of a sensitive indicator strain (Chandler, Davidson, Long, and Monnier, 1951; El Ghoroury, 1952). The second method was to grow the test organism in a suitable culture fluid (Duthie, 1944) with penicillin inducement or on nutrient agar (Harper, 1943). Various chemical and physical processes were then used in attempts to concentrate and purify the enzyme.

Widely differing views on penicillinase activity were propounded as a result of these techniques. Kirby (1945) and later Spink and Ferris (1947) were unable to demonstrate that staphylococcal penicillinase had passed into fluid culture from the cell Received for publication 9 August 1962. body. They believed it to be entirely endocellular. Bondi and Dietz (1944) found no diffusible penicillinase in five strains of Staph. aureus. These strains may have all been sensitive to penicillin, because, in 1945 , the same workers reported that 16 naturally resistant strains all produced penicillinase. None of 99 sensitive strains of Staph. albus and aureus produced detectable diffusible penicillinase. Gots' (1945) results with a more sensitive technique were essentially similar. Later work in many quarters gave various explanations for these divergent views. It was found that the enzyme is largely adsorbed on passage through Seitz filter pads (Duthie, 1944; Lepage, Morgan, and Campbell, 1946) or candles. Similar adsorption may occur on passing it through fritted glass filters. Penicillinase has a marked affinity for glass surfaces (Pollock, 1957), so that glass beads or abrasives used to destroy cells might well remove it almost entirely (Brunner, Kraushaar, and Prohaska, 1959-60). Froth from vigorous shaking can adsorb much of the enzyme, which is inactivated or inhibited by traces of anionic surface active agents.

These difficulties were overcome as they became known, and increasingly complex and fruitful methods were published; the method of Pollock, Torriani, and Tridgell (1956) gives a prolific yield of penicillinase from a $B$. cereus mutant; they were thereby able to elucidate most of the properties of the crystalline product. For assay of the enzyme, produced on this scale, manometric and iodometric methods were developed but these are insensitive and 
unsuited to small-scale production (Wolff and Hamburger, 1962).

With the advent of the newer penicillins during the last three years, and the comparative immunity of some of them to destruction by penicillinase, it has become increasingly necessary to find a simple yet sensitive screening method for the detection of diffusible penicillinase from staphylococci. It was therefore decided to follow up observations by Smith and Smith (1945) that penicillinase could diffuse freely through a gradocol membrane without loss of potency; these findings were amply confirmed in later papers, notably that by Eriksen and Hansen (1954).

The present paper gives fuller details of the method which we referred to in an earlier publication (Stewart, Coles, Nixon, and Holt, 1961), together with an assessment of penicillinase produced by staphylococci, as assayed by this more sensitive technique. A similar method, incorporating an indicator for the detection of penicilloic acid, has recently been developed independently by Knox and Smith (1951). Our objective in studying penicillinase is primarily concerned with the breakdown of the newer penicillins, and we are therefore also describing applications of the membrane technique for this purpose.

\section{METHOD}

MATERIAL AND MEDIA Throughout the series of experiments outlined below, Oxoid membrane filters were used. These are permeable membranes of cellulose acetate made by Courtaulds, which permit free diffusion of particles up to molecular weight of 50,000. Penicillinase from $B$. cereus is reported to have a molecular weight of about 35,000 , and although no definite reports of the molecular weight of staphylococcal penicillinase have been found, we felt it reasonable to assume that, should it differ structurally from other penicillinases, it would be of the same order of size. Membranes of 5 or $6 \mathrm{~cm}$. diameter are most suitable with a printed grid on one side which is helpful fcr location purposes. For sterilization, the membranes are immersed in infusion broth in a $250 \mathrm{ml}$. beaker. A circular weight is placed over the pile of membranes and the beaker put into a boiling water bath for about 20 to 30 minutes. The innermost membrane reaches a temperature of $95^{\circ} \mathrm{C}$. within a few minutes. Several hundred membranes have been prepared by this method and no preparations have been contaminated. The method ensures that the membrane is permeated with nutrient fluid to initiate rapid growth of test organisms and facilitate diffusion of exocellular metabolites. After the broth has cooled to about $70^{\circ} \mathrm{C}$. the membranes are lifted with sterile dissecting forceps and placed directly on to an agar base in $3 \frac{1}{2}$ in. petri dishes. With practice it is possible to lay the membrane quite flat on the agar surface without any trapped air, which upsets diffusion. The plates must be dried face downward for one hour at $37^{\circ} \mathrm{C}$.
Infusion broth agar, as used for penicillin assay, and infusion broth, both obtained from the Southern Group Laboratory, were used throughout.

Commercial penicillinase was obtained from $\overrightarrow{\vec{A}}$ Burroughs, Wellcome and Co., in the form of Neutrapen, a high-potency purified cereus penicillinase. This is? supplied in crystalline form, and, reconstituted at 400,000 units $/ \mathrm{ml}$., served as a reference standard.

EXPERIMENTAL RESULTS Preliminary experiments showed that, during overnight incubation, commercial penicil- linase at concentrations of 10 and 100 units $/ \mathrm{ml}$. diffused $P$ through the sterilized membrane into an agar base $\vec{\overrightarrow{ }}$ incorporating 1 to $10 \mu \mathrm{g} . / \mathrm{ml}$. penicillin G. Areas of $\mathrm{o}^{\omega}$ penicillin $\mathrm{G}$ inactivation were clearly demonstrated by flooding the plate with a suspension of penicillin G-? sensitive organisms (see later). After overnight incubation, $\vec{\sigma}$ the indicator organism grew freely in inactivated areas. $\omega$ This appeared so promising that it was decided to employ N the well-founded observation that penicillin $G$ attacks $\omega$ only the actively growing bacterial cell and has no을 effect on the cell in the resting phase (Hobby, Meyer, and Chaffee, 1942; Rammelkamp and Keefer, 1943). It was 3 found that a penicillin G-sensitive indicator organism could be incorporated in an agar base, together with penicillin $G$, at such a concentration that the indicator $\mathscr{\odot}$ organism was held in the resting phase in the agar base, $\omega$ and the organism under test for penicillinase production grew freely on the membrane. Thus penicillinase produced by the test organism released circumscribed areas of indicator growth. Furthermore, the presence of subinhibitory concentrations of penicillin $G$ induced $\frac{\otimes}{\not}$ production of penicillinase by the test organism, as in the $\mathscr{D}$ modified Gots' test described by Haight and Finland $\overrightarrow{\vec{O}}$ (1952).

INDICATOR ORGANISMS Different organisms were incorporated in agar plates containing penicillin $\mathbf{G}$ in concentrations of 0 to $0.01,0.05$ to 0.1 , and 0.5 to $1.0 \mu \mathrm{g}$. $/ \mathrm{ml}$. Experiments with varying inocula showed that a sowing into $20 \mathrm{ml}$. agar of $0.2 \mathrm{ml}$. of a broth suspension of 3 indicator organism, standardized at $1 \times 10^{8}$ organisms $/$. ml., gave light but evenly distributed colonial pavement growth. The Oxford staphylococcus (N.C.T.C. 6571) was usually suitable as an indicator organism, as it was found $\frac{\text { 의 }}{2}$ to be stable in behaviour and, when released from penicil- $\rightarrow$ lin G suppression, grew more rapidly. In a few experiments where less active organisms were tested for production of penicillinase, a group A Streptococcus was incorporated 0 in a blood agar base; growth of this indicator was accen- $N$ tuated by zones of haemolysis, provided that no soluble N haemolysins were produced by the test organism itself. Sarcina lutea was also used in confirmatory experiments, as it grew at $25^{\circ}$, and plates did not have to be incubated $\stackrel{\circ}{\circ}$ a second time.

Penicillin G concentration in base Penicillin $G$ in 7 concentrations ranging from 1.0 to $20.0 \mu \mathrm{g} . / \mathrm{ml}$. was $\bar{O}$ incorporated in a series of agar plates, on which mem- $\mathbb{D}$ branes were layered and inoculated heavily, in spots, $\frac{\rho}{\mathbb{D}}$ with fresh clinical isolates of Staph. aureus of widely $\varrho$ varying sensitivity to penicillin $\mathrm{G}(0.01 \mu \mathrm{g} . / \mathrm{ml}$. to $100 \mu \mathrm{g} . / 2$ 


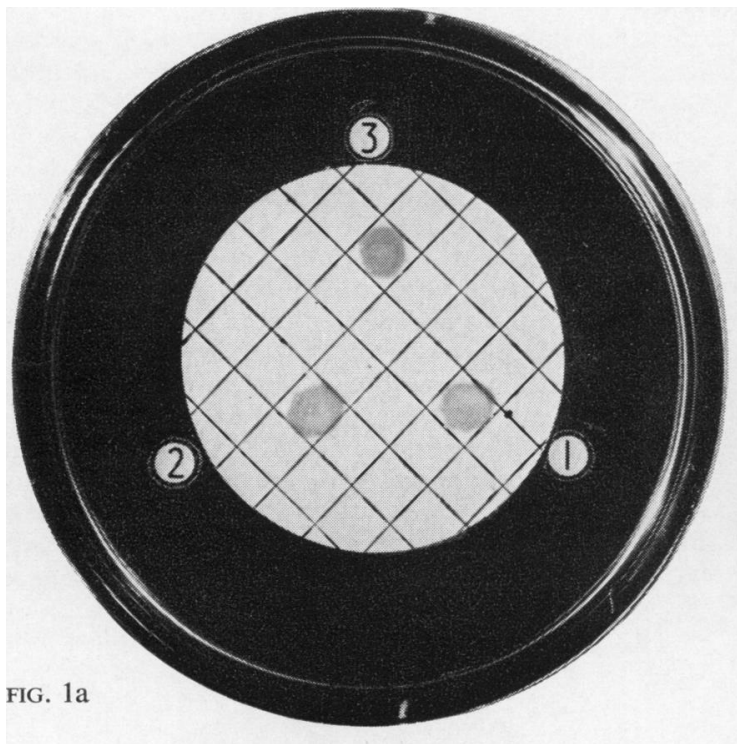

FIG. 1. One-stage membrane technique for detection of penicillinase.

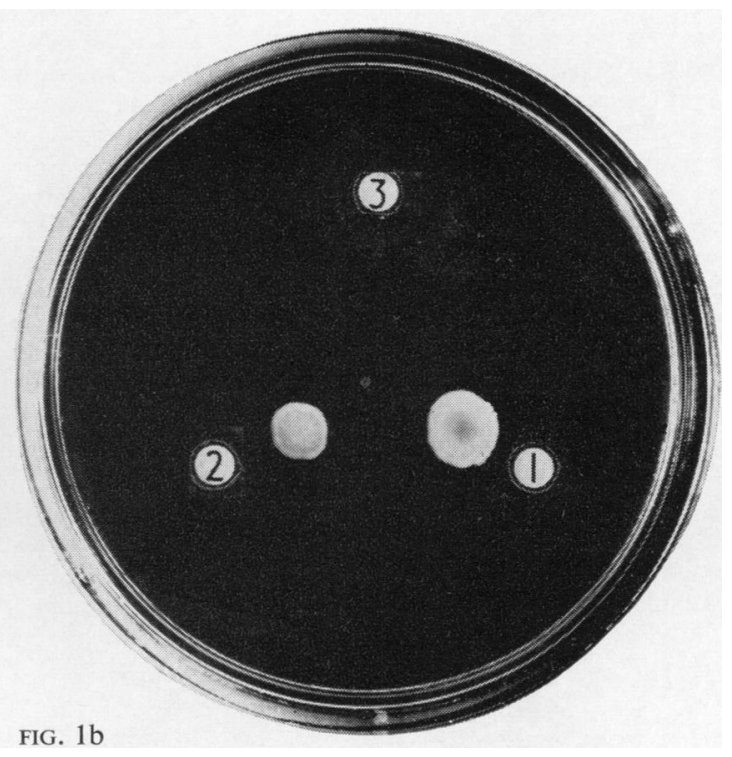

FIG. $1 \mathrm{~b}$ ml.). The highest concentration at which all strains grew freely on the membrane was $2 \mu \mathrm{g}$. $/ \mathrm{ml}$. penicillin G. This level was adopted for routine purposes. Since penicillinsensitive indicator strains, such as the Oxford staphylococcus, are unable to grow within plates containing more than $0.05 \mu \mathrm{g} . / \mathrm{ml}$. penicillin $\mathrm{G}$, their release by penicillinase and subsequent growth indicates that the local concentration of penicillin $G$ has been reduced below this level (Fig. 1).

STORAGE OF SEEDED PLATES Nutrient agar plates, containing $2 \mu \mathrm{g} . / \mathrm{ml}$. seeded with Oxford staphylococcus and layered with membranes, were stored in air-tight polythene bags at $4^{\circ} \mathrm{C}$., $20^{\circ} \mathrm{C}$., and $37^{\circ} \mathrm{C}$. At regular intervals a spot inoculum of a known active penisillinaseproducing Staph. aureus was made; after incubation the membrane was removed and the plate re-incubated to check indicator growth, indicative of destruction of the penicillin in the agar.
The results (Table I) indicate that a sensitive organism can be held in the resting phase under penicillin $G$ suppression for several weeks at $4^{\circ} \mathrm{C}$. but not at $37^{\circ} \mathrm{C}$. The indicator growth at the end of four weeks was just as prolific as at the beginning; this in itself suggests a means of storing a penicillin-sensitive organism indefinitely at $4^{\circ} \mathrm{C}$., to be released with penicillinase whenever required.

\section{ADAPTATION OF METHOD FOR TESTING SPREADING OR SWARMING BACTERIA}

Experiments with coliform/aerogenes bacteria, and also with strains of Proteus, showed that it was difficult to obtain clear-cut detection of penicillinase production by these. No matter how carefully the membrane plates were dried, sufficient moisture remained to permit spreading or swarming of mobile members of these groups. The addition of any of the customary anti-swarming agents, e.g., bile salts, chloral hydrate, dispersol, tended to alter

TABLE I

GROWTH OF OXFORD STAPH. AUREUS (N.C.T.C. 6571) AS INDICATOR ORGANISM IN PLATES EXPOSED TO PENCILLINASE-FORMING STAPHYLOCOCCI ON MEMBRANES AT VARIOUS INIERVALS

Temperature of Storage

\begin{tabular}{|c|c|c|c|c|c|c|}
\hline \multirow{2}{*}{$\begin{array}{l}\text { Temperature of Storage } \\
\left({ }^{\circ} \mathrm{C} .\right)\end{array}$} & \multicolumn{6}{|c|}{ Indicator Growth after Storage } \\
\hline & 0 & 24 Hours & 2 Days & 7 Days & 14 Days & 23 Days \\
\hline $\begin{array}{r}4 \\
22 \\
37\end{array}$ & $\begin{array}{l}++ \\
++ \\
++\end{array}$ & $\begin{array}{l}++ \\
++ \\
0\end{array}$ & $\begin{array}{l}++ \\
++ \\
0\end{array}$ & $\begin{array}{l}++ \\
++ \\
0^{+}\end{array}$ & $\begin{array}{l}++ \\
0\end{array}$ & $\begin{array}{l}++ \\
0\end{array}$ \\
\hline
\end{tabular}


the pattern of growth, and therefore invalidated assessment of penicillinase production. Membrane plates were therefore prepared from $4 \%$ and $6 \%$ agar incorporating penicillin $\mathrm{G}$ as above at $2 \mu \mathrm{g}$. $/ \mathrm{ml}$. The Oxford staphylococcus was used as indicator. A range of organisms was tested for penicillinase production on these plates. Staph. aureus gave excellent, but slightly compressed, zones of indicator growth. At both $4 \%$ and $6 \%$ coliform/ aerogenes bacilli, Proteus, and enterococci all gave restricted primary growth but, where penicillinase was formed, the resultant indicator growth was clearly observed. When testing these organisms, 4\% agar has now been adopted as routine.

\section{ADAPTATION OF THE METHOD TO THE NEWER PENICILLINS}

The validity of the above method obviously depends upon the survival of a proportion of the indicator organisms in the penicillin-loaded agar. This occurred
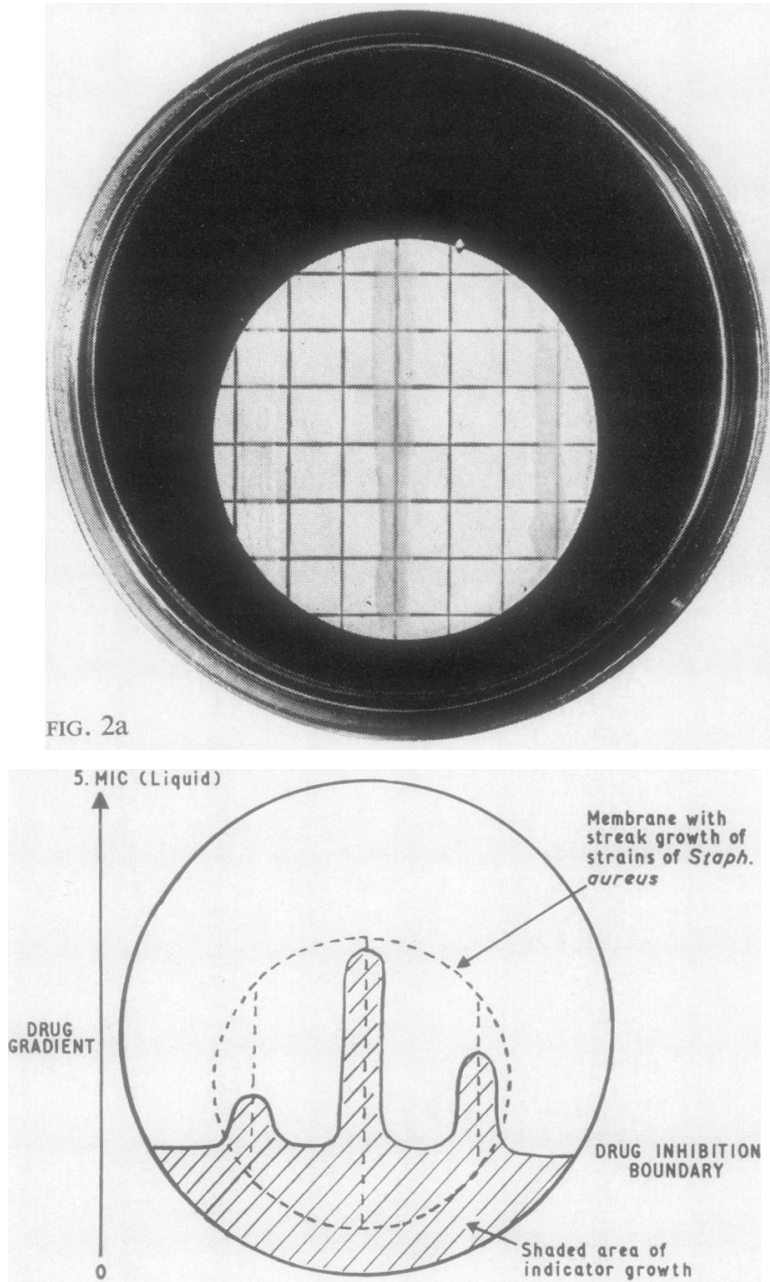

FIG. $2 \mathrm{c}$ when penicillin $G$ was used under the conditions described, but not necessarily with methicillin and other newer penicillins which were, at appropriate concentrations, completely bactericidal to organisms even in the resting state in agar stored at $4^{\circ} \mathrm{C}$. To detect destruction $\stackrel{\oplus}{\rightarrow}$ or degradation of these penicillins, it was therefore $\bar{C}$ necessary to apply the method in two stages; instead of $\overline{\bar{\sigma}}$ seeding the indicator organism into the drug-loaded agar $\frac{\bar{\omega}}{\partial}$ at the outset, the plate was incubated with the membrane $\mathbb{\Phi}$ and test organisms and the indicator organism flooded $\frac{2}{2}$ over the surface after removal of the membrane. This $\infty$ two-stage technique was more liable to occasional con- $\overrightarrow{0}$ tamination since the indicator organism was poured over $\vec{A}$ agar which had already been incubated. For the routine $\vec{\omega}$ detection of penicillinase, with penicillin $\mathbf{G}$ as the substrate, the one-stage method was therefore preferable. $\frac{8}{0}$ Both methods were suitable for detecting anti-penicillin $\overrightarrow{\vec{T}}$ factors in lysates, filtrates, dialysates, and crude cultures of test organisms.

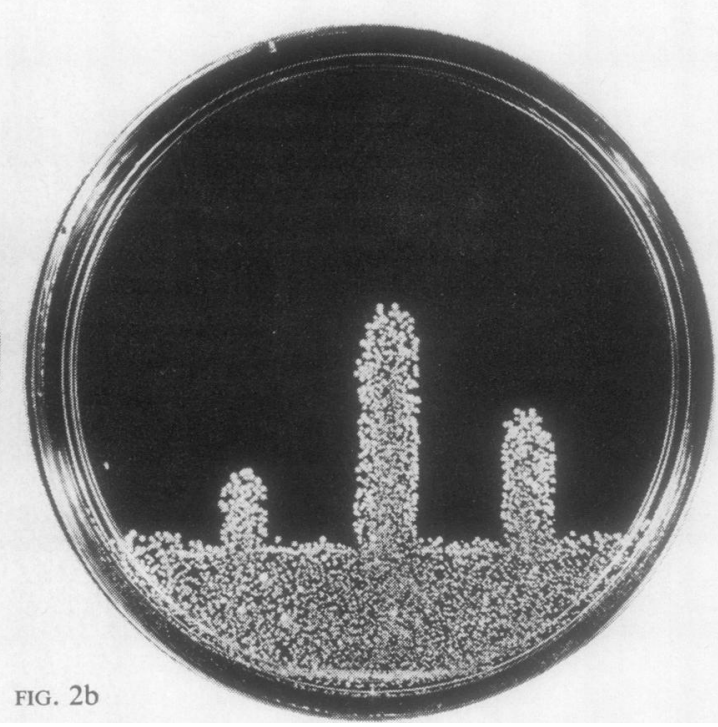

品

FIG. 2. Two-stage membrane technique with gradient $\mathrm{N}$ plate, for detection of traces of penicillinase.

2a Streak growth of three strains of Staph. aureus afterc $24 \mathrm{hr}$. at $37^{\circ} \mathrm{C}$. on membrane layered over agar with penicillin $G$ gradient $(0$ to $0.1 \mu \mathrm{g} . / \mathrm{ml}$.).

2b Membrane removed, indicator organism inoculated by flooding. Growth of indicator beyond the boundary inhibiting the drug. The central Staph. aureus has inactivated penicillin $G$ along the gradient for the whole length of its growth, while the others show lesser degrees of inactivation. $\frac{\text { Pे }}{10}$

2c Explanatory diagram of above. 
MODIFICATION OF THE METHOD FOR GRADIENT PLATES

The techniques described above are suitable for qualitative and rough quantitative estimates of the formation of penicillinase. For more accurate quantitative estimates, for comparative purposes, and for the detection of marginal destruction of a penicillin, the following method was devised.

Antibiotic gradient plates were prepared by successively pouring two superimposed wedges of agar. The lower wedge contained no antibiotic. The upper wedge incorporated the particular penicillin at a suitable level previously found to be five times the minimum inhibitory concentra.ion in liquid media. These plates, after floodseeding with a dilute suspension of the indicator organism (about $2 \times 10^{7}$ organisms $/ \mathrm{ml}$.), gave on incubation a reasonably well-defined limit of growth about one-third the distance up the gradient.

In practice gradient plates were poured as described and a membrane disc was layered; heavy streak inocula of test organisms were applied at right angles to the anticipated line of indicator inhibition (Fig. 2a). After overnight incubation the membrane was removed, the plate flooded with a suspension of indicator organisms and reincubated. The slightest inactivation or degradation of the penicillin by the test organism or its diffusible products was clearly shown by a peak of indicator growth extending up the concentration gradient, beyond the normal limit of growth (Fig. $2 b$ and c).

The object of these experiments was to detect inactivation of various penicillins and related substances to levels at which therapeutic activity was lost. We are not therefore in this text differentiating between the two main forms of penicillinase, i.e., $\beta$-lactamase and amidase. It can be assumed that inactivation by staphylococci is due to $\beta$-lactamase, but many of the coliforms tested possessed amidase as well as, or instead of, $\beta$-lactamase, and inactivated penicillin by this enzyme.

\section{REFERENCES}

Abraham, E. P., and Chain, E. (1940). Nature (Lond.), 146, 837.

Bondi, A. Jr., and Dietz, C. C. (1944). Proc. Soc. exp. Biol. (N.Y.), $56,132$.

Brunner, R., Kraushaar, A., and Prohaska, E. (1959-60). Antibiot. Ann., 7, 169.

Chandler, C. A., Davidson, V. Z., Long, P. H., and Monnier, J. J. (1951). Bull Johns Hopk. Hosp., 89, 81.

Duthie, E. S. (1944). Brit. J. exp. Path., 25, 96.

El Ghoroury, A. A. A. (1952). Science, 115, 20.

Eriksen, K. R., and Hansen, D. (1954). Acta path. microbiol. scand., $35,169$.

Gots, J. S. (1945). Proc. Soc. exp. Biol. (N.Y.), 60, 165.

Haight, T. H., and Finland, M. (1952). Amer. J. clin. Path., 22, 806.

Harper, G. J. (1943). Lancet, 2, 569.

Hobby, G. L., Meyer, K., and Chaffee, E. (1942). Proc. Soc. exp. Biol. (N.Y.), 50, 277.

Kirby, W. M. M. (1945). J. clin. Invest., 24, 170.

Knox, R., and Smith, J. T. (1961). Nature (Lond.), 191, 926.

Lepage, G. A., Morgan, J. F., and Campbell, M. E. (1946). J. biol. Chem., 166, 465.

Pollock, M. R. (1957). J. Pharm. Pharmacol., 9, 609.

-, Torriani, A.-M., and Tridgell, E. J. (1956). Biochem. J., 62, 387.

Rammelkamp, C. H., and Keefer, C. S. (1943). J. clin. Invest., 22, 425.

Smith, W., and Smith, M. M. (1945). Lancet, 1, 809.

Spink, W. W., and Ferris, V. (1947). J. clin. Invest., 26, 379.

Stewart, G. T., Coles, H. M. T., Nixon, H. H., and Holt, R. J. (1961). Brit. med. J., 2, 200.

Wolf, D. A., and Hamburger, M. (1962). J. Lab. clin. Med., 59, 469. 